\title{
Research on quality state perception of digital production line in aviation enterprise
}

\author{
You Yingchao ${ }^{1, a}$, Duan Guijiang ${ }^{1}$, Qu Xinhe \\ ${ }^{1}$ School of Mechanical Engineering and Automation, Beihang University, Beijing,100191, China \\ ${ }^{2}$ Changhe Aviation Industrial(Group) Co.,LTD., Jingdezhen,333000, China
}

\begin{abstract}
With the development of intelligent manufacturing technology, the requirements of production process management have been improved. In view of the difficulty of the production line of a certain enterprise, the quality state perception frame and technical scheme of digital production line are put forward. and the key information types in the production line are combed.This paper presents a multi-source heterogeneous data model for the production process. Finally, the state-sensing system of digital production line is developed and applied successfully in an aviation enterprise.
\end{abstract}

\section{Introduction}

With the development of intelligent manufacturing technology, the influence of intelligent manufacturing technology on the manufacturing field is becoming more and more deep. The intelligent, networked, flexible and green manufacturing process has become the main direction of the manufacturing industry. Intelligent manufacturing technology, such as mobile communication network, data sensing monitoring, information interaction integration and advanced artificial intelligence, provides more effective technical support for automatic production, fault identification and intelligent decision making in intelligent factory. The acquisition of state information in production process is the first step in the process of intelligent manufacturing, and at the same time, it provides guarantee for data analysis and production process management. At present, there are problems in production workshop, such as opaque information in production process, low integration of production information and backward management. Workshop managers can not get timely information about the production process status.

In recent years, experts and scholars at home and abroad have carried out a lot of research and Practice on the status perception of digital workshops. For example, Abrishamkar ${ }^{[1]}$ put forward a framework of remote monitoring and manufacturing system based on TCP/IP protocol for low automation manufacturing industry. The information of the workshop is visualized. Senkuvien et al. ${ }^{[2]}$ discussed the method of real-time production schedule monitoring and real-time monitoring of the workload of the equipment. Practice has proved that the method proposed is effective. Harrison ${ }^{[3]}$ realize the information interaction within the factory by deploying the RFID network and storing product manufacturing information in the RFID tag. Liu Dezhi ${ }^{[4]}$ combines the bar code recognition technology with the ERP system, realizes the real-time management and control of the workshop site through the fast acquisition of field data, and puts forward an industrial control solution based on RFID technology. HeRui ${ }^{[5]}$ uses Sun Java development language to design and develop a monitoring screen generation software with the function of the integrated graphics library, which solves the problem of sharing graphic data..

The above research has done a lot of valuable achievements in the visualization and real-time monitoring of production status information in digital workshop, and promoted the construction of intelligent factory. However, there are still some shortcomings in the above research. The information about the quality state of production line parts is mentioned less, and the research on information integration in digital workshop is not enough. Therefore, in this paper, by using the existing research results and using web, WebService and other technologies, a technical scheme of state perception of digital production line is proposed. The information of human, machine, material, method and ring state in the production line is acquired automatically and integrated into graphical display through information processing. The quality state perception technology of the digital production line provides technical support for the dynamic scheduling of production planning, enhances the interaction between the workshop manufacturing information and the plan management information, and provides the manager's quick response to the production plan and provides decision support. In the form of graphics and video, the manufacturing process is restored and visual management is strengthened. It has been applied to an aviation manufacturing enterprise, which verifies the feasibility and effectiveness of the scheme. 


\section{Multisource, heterogeneous, ubiquito us quality state perception model}

The manufacturing process of digital production line is characterized by discretization of processing equipment, diversified product varieties and heterogeneous data sources. With all-round, multi-level production line within the data, we demand analysis about the production state of each link in the process of producing, determine the product manufacturing process to collect the data types and specific requirements. From the four dimensions of personnel, equipment, products and environment, the multi-source state perception model of digital production line is shown in Figure 1

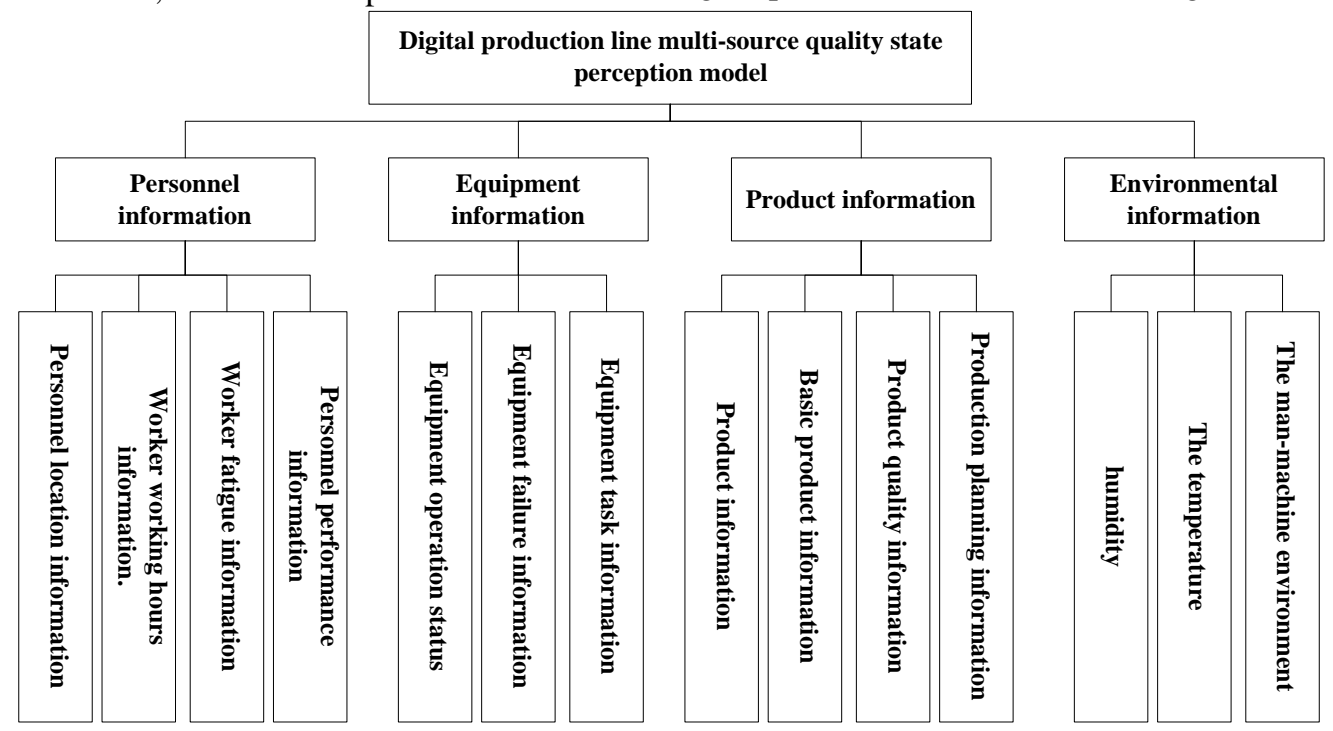

Figure 1 Digital production line multi-source quality state perception model

Equipment information: mainly includes the basic information of equipment and operation state information. The basic information of the equipment mainly includes the ID number of the equipment, device model, equipment name, equipment parameters, equipment performance and static information of the device in position. The operation state of the equipment mainly includes equipment outage, equipment processing and equipment failure. The state information of machine tool includes the spindle speed of machine tool, feed speed, tool, fixture and so on.

Product information: product information mainly includes product basic information and product quality information. The basic information includes the processing station, state information, physical quantity, quality data and related equipment information of the products. Quality information includes: product testing data, super - difference information, product characteristic information, etc.

Personnel information: Personnel information mainly include personnel attendance, personnel location, working hours information, performance information, fatigue information, proficiency information. Personnel location information refers to the information of personnel and swimming personnel involved in processing parts and equipment during production. Time information refers to the time a worker has to finish at the beginning of a task. The measure of proficiency information is the ratio of worker time information to standard time information.

Environmental information: environment refers to the space in which a worker completes a task. Environmental information includes: temperature, humidity, man-machine environment. Through access to information environment, the human - machine environment system as the research object, utilizing the knowledge of physiology, psychology and other related disciplines, according to the conditions and characteristics of humans and machines, rational distribution and machine operating functions, and make it adapt to each other, so as to create a comfortable and safe working environment, make the efficiency to achieve the optimal.

\section{State perception framework and techn ical of scheme of digital production line}

Traditional production lines have difficulties in product management, poor material delivery, poor real-time information and poor product quality. In order to solve the problems existing in the production process, realtime and effective data collection is carried out to achieve the purpose of state perception. In this section, by analyzing the information frame of digital production line, the general frame of state perception of digital production line is established. Framework accurately describes the manufacturing process of the change of information, namely, data collection, information processing, production process management and data feedback loop process, and contains the scene of the production data acquisition and PDM and ERP system manufacturing process such as the link between the supporting software and dependencies.

Through comprehensive application of RFID theory, MBD model carrier, webservice technology, multidimensional data fusion technology and sensor technology, data resources, including production personnel, production equipment, production of the real- 
time, are acquired and located in real time. With the support of the production process system, multi-source and heterogeneous data are expressed and stored in the database. The real-time data collected in the manufacturing process is uploaded to the information network, which is integrated with PDM and ERP system information, then the multi-source heterogeneous data model is established. Multiple heterogeneous data model with the support of function layer, the SPC analysis,
FEMA analysis, failure prediction analysis, and graphical display, manufacturing process control and production process for the enterprise management, quality control, to provide basic data in order to make quick decisions.

\subsection{Overall framework of state perception of digital production line.}

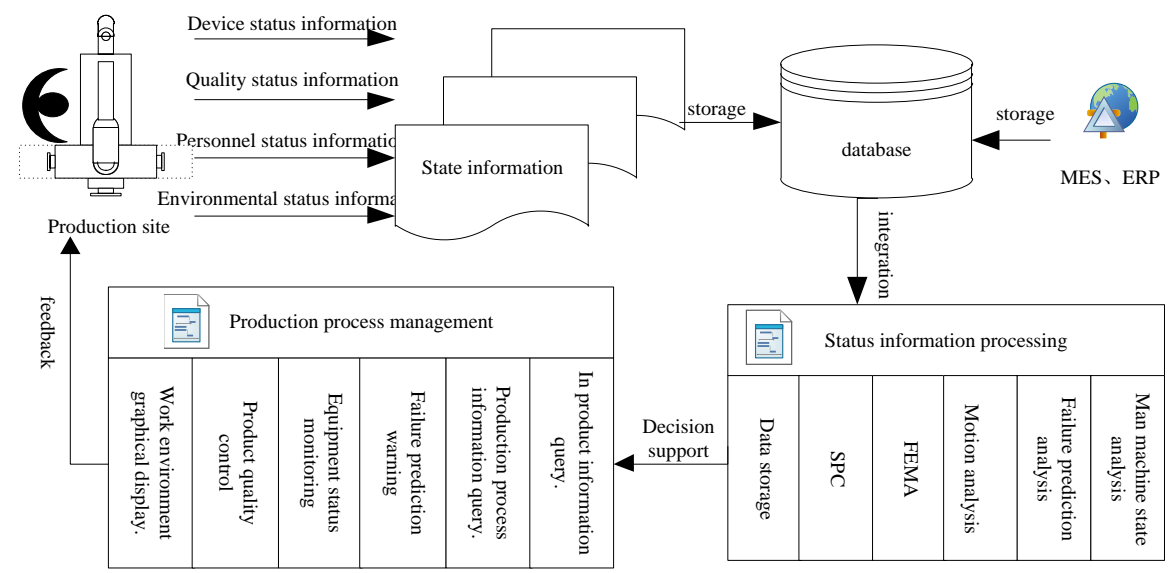

Figure 2 Digital production line quality state perception overall framework

As shown is the Figure 3, the general framework of state perception of digital production line includes four modules, production field data collection, multi-source data fusion, state information processing, and production process management module. The production field data collection includes equipment state information collection, quality state information collection, personnel state information collection and environmental state information collection; Multi-source data fusion module includes MES, ERP product information and real-time data integration and integration; State information processing includes: data storage, SPC analysis, FEMA analysis, action analysis, fault prediction analysis, and man-machine state analysis; The production process management module includes: work environment graphical display, product quality control, equipment state monitoring, fault warning, production process information query, and product information query. The logic relation between modules: the production field data acquisition module gathers the multi-dimensional data in the manufacturing process, and saves it to the database in a structured way, which is the basic link of state perception. The multi-source data fusion will combine the real-time data of production and the basic information of the product to prepare for the data analysis. The state information processing module processes the data and converts it into valuable information to provide technical support for the production process decision.

\subsection{State perception technology of digital production line}

The target of the digital production line state perception technology, whose principle is multivariate and ubiquitous, is solve the problem of lack of information collection and low degree of integration in the production line, which fully combines the key technology of the Internet of things. The technology solution is divided into three levels: state aware layer, data layer, application layer.

State awareness layer: First, the application of RFID to capture the dynamic information of the WIP, the RFID read and write interface can track the object with the logo, and the data structure is simple, can quickly and accurately reflect the quantity and position information of the object. This information can accurately reflect the working state of the product, the position information of the personnel, and the staff members, time information and so on. Through comprehensive testing information of WIP and parts processing information, First of all, the workers move the three coordinate machine measurements from the machine to the computer by using the application of web service technology. Next, the system interprets the report as structured data. Third, the system combines the structured data with the detection information of the manual acquisition equipment and forms the information of the product quality state. The product quality state information has played an important role to control the quality. The state information extraction of machine tool can be used to add macro instructions in NC files to extract the basic information and running state information of the machine tool. The working environment information can be transmitted to the database through the temperature and humidity sensor and the sound sensor. 
Data layer: data layer is the core component of multisource heterogeneous information integration model, composed by the personnel state module, equipment management module, environment state module, product quality module and multi-source heterogeneous information module of five parts. Multi-source heterogeneous information modules using pattern matching or mapping rule based information in a logical layer of workshop manufacturing process will be expressing forms, which can provide unified format different sources of information system . Through mapping and information service encapsulation, the system realizes the integration of functional layer and data layer with production equipment, product information and operator information., in the product information and the integration of operation personnel information, which can satisfy the business enterprise internal management of heterogeneous systems and the needs of different customers. The production line state sensing module and MES module carry out information integration in the data layer, and the product state information in MES is called by the state sensing module.

Functional layer: Based on data layer structured data, the data is analyzed and processed, then the internal product information, equipment state and environment of the workshop are presented to the manager in a threedimensional visual way. At the same time, the module provides a query interface for the product, equipment, environment and personnel state, providing the manager with decision support for the production process decision. Functional modules support SPC analysis and FEMA analysis to provide technical support for product quality control and fault prediction.

\section{Conclusion}

The research on state perception of digital production line is an important part of intelligent factory, which plays an important role in improving the information transparency and information integration of the workshop. In order to improve the workshop production process management, manufacturing efficiency, we build the digital production line state based on the actual problem in aviation enterprise digital production line, technical framework. On the basis of the existing production process, we carry out information collection from four dimensions of equipment, product, personnel and environment. The multi-source and heterogeneous data model is sorted into structured data, and the production field data collection, multi-source data fusion, state information processing and production process management module are established. Finally, using RFID technology, iot technology and webservice technology, we established the production line state awareness system and developed the software system using B/S architecture. The enterprise adopts the digital production line state sensing system to improve the information integration of the production line and provide technical support for the production process control.

\section{References}

1. M Abrishamkar,M Hussein etc. Advanced Materials Research,845, 975-979,(2013)

2. I. Senkuvienè,K Jankauskas Kvietkauskas .Mechanics, 20 (1),99-107,( 2014)

3. Harrison M, Mcfarlane D,Parlikad A K,et al. IEEE International Conference on industrial informatics. IEEE ,2004,507-512,(2004)

4. D Liu etc,Modern Manufacturing Engineering, 2010 (5) :16-20,2010

5. R He etc,Industrial Control Computer, 19 (6) ,32-33, 2006 